\title{
Evaluation of PIG-A-mutated granulocytes and ex-vivo binucleated micronucleated lymphocytes frequencies after breast cancer radiotherapy in humans
}

\author{
Rémi M. Bonetto ${ }^{1}$ | Pierre Castel ${ }^{2}$ | Stéphane P. Robert ${ }^{3}$ | Virginie M. Tassistro ${ }^{2}$ | \\ Magalie Claeys-Bruno $^{2}$ | Michelle Sergent ${ }^{2}$ | Camille A. Delecourt ${ }^{2}$ | \\ Didier Cowen $^{1}$ | Xavier Carcopino ${ }^{2,4}$ | Thierry G. Orsière ${ }^{2}$
}

\author{
${ }^{1}$ Aix Marseille University, APHM, CHU \\ TIMONE, Service de Radiothérapie-Oncologie, \\ Marseille, France \\ ${ }^{2}$ Aix Marseille University, Avignon Université, \\ CNRS, IRD, IMBE, Marseille, France \\ ${ }^{3}$ Aix Marseille University, INSERM, INRA, \\ C2VN, AMUTICYT Core Facility, Faculté de \\ Pharmacie, Marseille, France \\ ${ }^{4}$ Aix Marseille University, APHM, CHU NORD, \\ Service de Gynécologie-Obstétrique, Marseille, \\ France

\section{Correspondence} \\ Thierry G. Orsière, Institut Méditerranéen de \\ Biodiversité et d'Ecologie marine et \\ continentale (IMBE), Equipe Biomarqueurs, \\ Environnement, Santé, Faculté de Sciences \\ Médicales et Paramédicales de la Timone, \\ 27 Bd Jean-Moulin, Marseille F-13385, Cedex \\ 05, France. \\ Email: thierry.orsiere@imbe.fr \\ Funding information \\ A*MIDEX, Grant/Award Number: ANR- \\ 11-IDEX-0001-02; ARS PACA, Grant/Award \\ Number: REVELA13; 20120658; SATT-SE, \\ Grant/Award Number: ID 870; French \\ National Research Agency, Grant/Award \\ Number: REVELA13
}

\begin{abstract}
Although the PIG-A gene mutation frequency (MF) is considered a good proxy to evaluate the somatic MF in animals, evidence remains scarce in humans. In this study, a granulocyte PIG-A-mutant assay was evaluated in patients undergoing radiation therapy (RT) for breast cancer. Breast cancer patients undergoing adjuvant RT were prospectively enrolled. RT involved the whole breast, with (WBNRT) or without (WBRT) nodal area irradiation. Blood samples were obtained from participants before (TO) RT, and T1, T2, and T3 samples were collected 3 weeks after the initiation of RT, at the end of RT, and at least 10 weeks after RT discontinuation, respectively. The MF was assessed using a flow cytometry protocol identifying PIG-A-mutant granulocytes. Cytokinesis-blocked micronucleated lymphocyte (CBML) frequencies were also evaluated. Thirty patients were included, and five of them had received chemotherapy prior to RT. The mean $( \pm S D)$ PIG-A MFs were $7.7( \pm 12.1)$ per million at T0, $5.2( \pm 8.6)$ at T1, $6.4( \pm 8.0)$ at T2 and $3.8( \pm 36.0)$ at T3. No statistically significant increases were observed between the PIG-A MF at TO and the MFs at other times. RT significantly increased the CBML frequencies: $7.9 \%$ \% $( \pm 3.1 \%$ ) versus $33.6 \%$ 。 $( \pm 17.2 \%$ ) $(p<.0001)$. By multivariate analysis, the CBML frequency was correlated with age at RT initiation ( $p=.043$ ) and irradiation volume at RT discontinuation ( $p=.0001$ ) but not with chemotherapy. RT for breast cancer therapy failed to induce an increase in the PIG-A MF. The PIG-A assay in humans needs further evaluation, in various genotoxic exposures and including various circulating human cells.
\end{abstract}

KEYWORDS

granulocyte, human, micronucleus assay, mutation, PIG-A gene, radiotherapy

\section{INTRODUCTION}

Genotoxic exposure has been identified as a potential cause of cancer due to induced DNA damage with variable consequences, depending on the individual (Houtgraaf et al., 2006). Until recently, genetic toxicology tests and methods of human gene mutation detection were not suitable for human biomonitoring studies. However, in the last months, PIG-A gene mutation has been used as a genotoxicity biomarker in various exposure scenarios in humans, in combination with the formation of micronuclei (Cao et al., 2020a, 2020b). Moreover, 
optimized protocols related to human blood PIG-A mutation and micronucleated reticulocyte flow cytometric assays have been published (Torous et al., 2020). This highlights the importance of developing tests that can quantify DNA damage in humans.

Research on the PIG-A gene as a genotoxicity biomarker has emerged with studies on paroxysmal nocturnal hemoglobinuria ( $\mathrm{PNH}$ ) (Takeda et al., 1993). The enzyme encoded by the PIG-A gene is critical for the biosynthesis of the membrane anchor glycosylphosphatidylinositol (GPI). Phenotypically, in PNH patients, PIG-A-mutant cells lack the GPIanchored complement inhibitor, leading to complement-mediated hemolysis and clinical features of the disease (Brodsky, 2014). In parallel, mutation-carrying blood cells and the corresponding phenotype have been identified in healthy individuals at extremely low frequencies (between 10 and $30 \mathrm{GPI}$-deficient cells per $10^{6}$ cells) (Araten et al., 1999; Hu et al., 2005). The PIG-A gene has therefore been considered a potential sentinel gene, being highly conserved among species (Kawagoe et al., 1994), with a mutation frequency (MF) reflecting the rate of acquired somatic mutations (Peruzzi et al., 2010). Reciprocity between a cell with a mutation at the PIG-A locus and a GPI-deficient phenotype has been recognized by the International Workgroup on Genotoxicity Testing (Gollapudi et al., 2015). Thus, it is now commonly accepted that GPIdeficient cells carry inactivating mutations within the PIG-A gene and are equally referred to as PIG-A mutants or PIG-A-mutated cells.

Using flow cytometry, several studies have enabled the development of the "PIG-A assay," which estimates the frequency of cells with a mutation in the PIG-A gene by measuring the frequency of GPI anchordeficient circulating cells (Kawagoe et al., 1994; Araten et al., 1999). Currently, this assay is commonly applied for genotoxicity testing in rodents. A significant increase in the Pig-a MF has been reported in rodent reticulocyte and erythrocyte populations following exposure to chemotherapeutic agents, such as $\mathrm{N}$-nitroso- $\mathrm{N}$-ethylurea and cisplatin (Miura et al., 2009; Phonethepswath et al., 2008, 2010; Dertinger et al., 2014 Bhalli et al., 2011), or $X$ and $\gamma$ ionizing radiation (Ohtani et al., 2012, 2014; Graupner et al., 2016). In humans, GPI-deficient cell identification is mainly performed for the diagnosis and monitoring of $\mathrm{PNH}$ patients (Parker et al., 2005; Borowitz et al., 2010). In relation to gene mutations, the MF of the PIG-A gene was found to be spontaneously higher not only in patients with cancer predisposition due to defects in DNA repair but also in cancer cells, suggesting the MF of the PIG-A gene as a potential biomarker of genomic instability (Olsen et al., 2017). A recent study reported higher frequencies of GPI-deficient erythrocytes in patients with esophageal adenocarcinoma than in healthy donors or patients with a preneoplastic status and a major increase in this population in patients receiving chemotherapy (Haboubi et al., 2019). However, data remain controversial, as such increases were not found in other studies on patients receiving different genotoxic chemotherapy regimens or on soldiers exposed to depleted uranium (Dobrovolsky et al., 2011; McDiarmid et al., 2011; Olsen et al., 2017).

The PIG-A assay has been evaluated in humans following ionizing radiation exposure. According to United Nations Scientific Committee on the Effects of Atomic Radiation (UNSCEAR) data, medical use of radiation accounts for $98 \%$ of the population dose contribution from all artificial sources and represents $20 \%$ of the total population exposure to ionizing radiation (UNEP, 2016). The UNSCEAR estimated that a 1-Gy exposure yields up to 40 DNA double-strand breaks, 1,000 DNA singlestrand breaks, and 500 damaged bases, including 150 DNA cross-links, per cell (United Nations, 2000). Radiation therapy (RT), as part of cancer treatment, is the most ionizing medical radiation modality. As breast cancer is the most common cancer in women worldwide, with some patients receiving chemotherapy in addition to RT, breast cancer patients are likely to be directly affected by radiation genotoxicity and therefore represent a suitable human model for the evaluation of the PIG-A assay in humans following ionizing radiation exposure.

The aim of this work was to prospectively evaluate the PIG-A assay by quantifying the GPI-deficient granulocyte frequency in women receiving $\mathrm{RT}$ for the treatment of breast cancer as a controlled genotoxic environment.

\section{2 | MATERIALS AND METHODS}

\section{1 | Patients}

Patients with recently diagnosed breast cancer were prospectively enrolled over an 18-month period from January 2016 to June 2017. Patients were included immediately after initial breast surgery, either conservative or radical (i.e., mastectomy). Only patients who were indicated with adjuvant RT were included. Patients with a history of previous RT were not included. Patients were differentiated according to the RT regimen they received: whole breast RT $(\mathrm{WBRT} ; n=14)$ and whole breast and nodal RT (WBNRT; $n=16$ ) (Figure 1). Relevant data on personal or familial medical history, smoking habits, alcohol consumption, drug intake, and recent $\mathrm{X}$-ray diagnostic examination were collected for each participant using a standard questionnaire prior to the first blood sampling time point. The protocol received approval from the Sud-Méditerranée 1 Committee for the Protection of Populations (ID RCB: 2015-A01753-46). Written informed consent was obtained from all participants.

\section{2 | Radiation therapy}

RT was delivered in three-dimensional conformational modalities (the usual technique recommended for adjuvant breast irradiation allowing a conformational beam for anatomic irradiation of the planning target volume consisting of the breast and lymph node area) with a Synergy accelerator (Elekta, Stockholm, Sweden), giving a dose rate set at $0.4 \mathrm{~Gy} / \mathrm{min}$. All patients received $50 \mathrm{~Gy}$ divided into 25 fractions of 2 Gy each. Patients who had a conservative surgery, that is, tumor removal only, received an additional overprint of $16 \mathrm{~Gy}$ divided into eight fractions. Patients who underwent total mastectomy, that is, removal of the whole mammary gland, received an additional overprint of $10 \mathrm{~Gy}$ divided into five fractions at the level of the scar, according to the risk of skin recurrence. Irradiation of the supraclavicular nodal area and the internal mammary chain was performed in patients with lymph node involvement or with tumors located in 
FIGURE 1 Flowchart of the population and sampling schedule. Among the 30 patients, 5 received chemotherapy before enrollment. CBML, cytokinesisblocked micronucleated lymphocytes assay; PIG-A, PIG-A assay; WBNRT, whole breast and nodal area radiation therapy; WBRT, whole breast radiation therapy

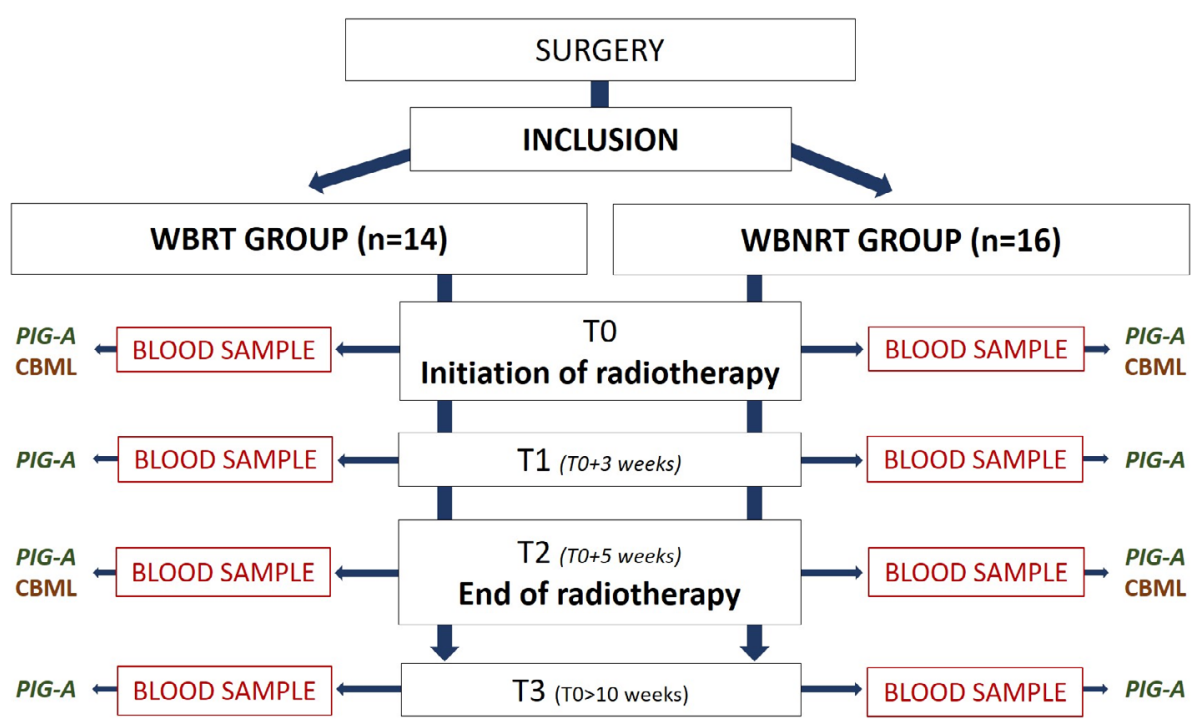

the inner part of the breast. Axillary irradiation was delivered in cases of axillary nodal macrometastasis when complementary nodal dissection had not been performed. For the internal mammary chain, the delivered dose was $50 \mathrm{~Gy}$ in 25 fractions of $2 \mathrm{~Gy}$ each in a combination of $20 \mathrm{~Gy}$ of photons and $30 \mathrm{~Gy}$ of electrons to spare mediastinal structures. For the supraclavicular and/or axillary area, the delivered dose was $46 \mathrm{~Gy}$ in 23 fractions of $2 \mathrm{~Gy}$. The irradiated bone volume was estimated with dosimetry calculations performed using a Philips Pinnacle treatment planning system (Philips Healthcare, Amsterdam, The Netherlands). For each participant, target volumes, organs at risk and the bone volume fraction within the $0.5 \mathrm{~Gy}$ isodose volume were contoured. The irradiated bone volume was calculated as an estimation of the volume of the irradiated bone marrow.

\section{3 | Blood samples}

Four blood samples were collected from each patient. The initial sample (TO) was obtained immediately before the initiation of RT. The second (T1), third (T2), and last (T3) samples were collected 3 weeks after the initiation of RT (within $2 \mathrm{hr}$ after the end of the last RT administration), at the end of RT, and at least 10 weeks after RT discontinuation, respectively. Blood samples were collected using anonymized EDTA tubes (Beckton-Dickinson vacutainer) for the PIG-A assay and lithium heparin tubes for the micronucleus assay. Tubes were immediately sent to the "Biogenotoxicology, Human Health \& Environment" Laboratory (IMBE-Mediterranean Institute of Biodiversity and EcologyMedicine School of Marseilles) for immediate processing.

\subsection{Quantification of the GPI-deficient granulocyte frequency: The PIG-A assay}

Two milliliters of anticoagulant-treated fresh blood was mixed with $20 \mathrm{ml}$ of erythrocyte lysis solution (154 mM ammonium chloride,
$10 \mathrm{mM}$ potassium hydrogen carbonate, and $0.1 \mathrm{mM}$ EDTA) for $10 \mathrm{~min}$ at room temperature. After centrifugation at $300 \mathrm{~g}$ for $5 \mathrm{~min}$ at room temperature, immunostaining was performed directly on the pellet by adding anti-CD15-PB, anti-CD33-PC7, anti-CD45-Krome Orange, and anti-CD66b-APC antibodies and FLAER-Alexa488 and incubating the mixture for $30 \mathrm{~min}$ in the dark at room temperature. The pellet was washed with PBS and resuspended in PBS before immediate flow cytometry analysis.

Quantification of PIG-A-mutant granulocytes was performed using flow cytometry. Fluorochrome-conjugated antibodies were chosen according to good laboratory practice recommendations for the diagnosis and monitoring of PNH patients. For selection, the antibodies were selected according to their ability to identify granulocytes within whole blood: anti-CD15-PB, anti-CD33-PC7, and anti-CD45-KO. Reagents used for the identification of GPIdeficient cells within the granulocyte population were an anti-CD66bAPC antibody, a GPI-dependent granulocyte-specific antibody, and FLAER-Alexa488, a GPI-specific marker.

Analyses were carried out on a Gallios model flow cytometer (Beckman-Coulter, Villepinte, France). The results are expressed using cytograms. The phenotyping protocol was as follows: first, cell doublets were eliminated using the forward scatter area versus forward scatter height parameters. The positivity of CD45 expression allowed us to focus the analysis on immune cell populations. In this framework, two graphs (CD45 vs. cell structure and then CD45 vs. CD33) made it possible to limit the analysis to nonlymphoid immune cell populations. Two independent GPI myeloid markers, CD15 and CD33, were used to limit the analysis to granulocytes. Selected granulocytes were defined as CD15+/CD33dim, and granulocyte selection was limited to the highest 95\% CD15 signal intensity to increase specificity. Finally, among these granulocytes, FLAER and CD66b doublenegative cells were considered mutants. The analysis was performed on at least $1 \times 10^{6}$ granulocytes, and the acquisition rate was limited to 4,000 events per second to improve the accuracy of the analysis. The primary endpoint was the MF, defined as the number of GPI-deficient 
granulocytes among all granulocytes, determined by flow cytometry and expressed per million events.

The first step of the optimization process consisted of defining the incubation time, antibody concentrations, and flow cytometric parameters (data not shown). Optimization and validation of GPIdeficient granulocyte detection were performed using blood samples from a healthy donor and a PNH patient. This allowed for the validation of the detection strategy using $\mathrm{PNH}$ cells as the positive control. The gating strategy is illustrated in Figure 2. The fluorescence thresholds for FLAER and CD66b below which an event was considered doubly negative were obtained using a blood sample from the $\mathrm{PNH}$ patient. The stability of the counting of GPI-deficient cells was confirmed by means of serial dilution producing a number of GPIdeficient cells lower than 50; this value is commonly considered a lower limit for flow cytometry (Figure S1).

\section{$2.5 \mid$ Micronucleus test}

The frequencies of cytokinesis-blocked micronucleated lymphocytes (CBMLs) in culture were determined for TO (before the initiation of radiotherapy) and $\mathrm{T} 2$ (at the end of radiotherapy, the mean delay between T0 and T2 was 212.4 [ \pm 84.2 ] days) samples. One aliquot of whole blood was transferred into flasks and incubated at $37^{\circ} \mathrm{C}$ with $5 \% \mathrm{CO} 2$ for $44 \mathrm{hr}$ in X-vivo 10 culture medium (Lonza S.p.r.l, Verviers, Belgium) supplemented with 1\% heparin (Sigma Aldrich, St. Quentin Fallavier, France) and 1\% phytohemagglutinin (Sigma Aldrich). The cells were then treated with cytochalasin B (Sigma Aldrich) and incubated for $24 \mathrm{hr}$ at $37^{\circ} \mathrm{C}$ with $5 \% \mathrm{CO} 2$. At the end of the incubation, the cells were centrifuged $\left(220 \mathrm{~g}, 10 \mathrm{~min}\right.$, at $\left.20^{\circ} \mathrm{C}\right)$, treated with $0.075 \mathrm{mM} \mathrm{KCl}$ and centrifuged again $\left(150 \mathrm{~g}, 1 \mathrm{~min}\right.$, at $20^{\circ} \mathrm{C}$ ).

The cells were then treated with fixative (methanol: acetic acid $3: 1 \mathrm{v} / \mathrm{v}$ ) for $20 \mathrm{~min}$ at $4^{\circ} \mathrm{C}$ and centrifuged ( $300 \mathrm{~g}, 10 \mathrm{~min}, 20^{\circ} \mathrm{C}$ ). The cell pellets were resuspended in fixative and stored at $4^{\circ} \mathrm{C}$. Lymphocytes in fresh fixative were dropped onto clean slides on ice, air-dried and stained with $2 \%$ Giemsa (Sigma Aldrich). Blinded and duplicate $\mathrm{MN}$ counts were performed by the same investigator on lymphocytes with well-preserved cytoplasm only. Two thousand binucleated (BN) cells were analyzed for each subject. Cells were cytologically scored using the cytome approach to evaluate the viability status (necrosis, apoptosis), mitotic status (mononucleated, BN, and multinucleated), and presence of micronuclei (Fenech, 2007). The cytokinesis-block proliferation index (CBPI) was calculated as follows: $\mathrm{CBPI}=(\mathrm{M} 1+2 \mathrm{M} 2+3 \mathrm{M} 3) / \mathrm{N}$ (where M1-M3 represent the numbers of cells with 1 to 3 or more nuclei and $N$ is the total number of viable cells scored, that is, total cell number excluding necrotic and apoptotic cells). The results are reported as CBMLs per 1,000 cells.

\subsection{Statistical analysis}

When appropriate, normality was assessed using the Kolmogorov-Smirnov test. Non-Gaussian data are presented as the median [interquartile range]. Corresponding means $( \pm S D s)$ are also provided for the PIG-A
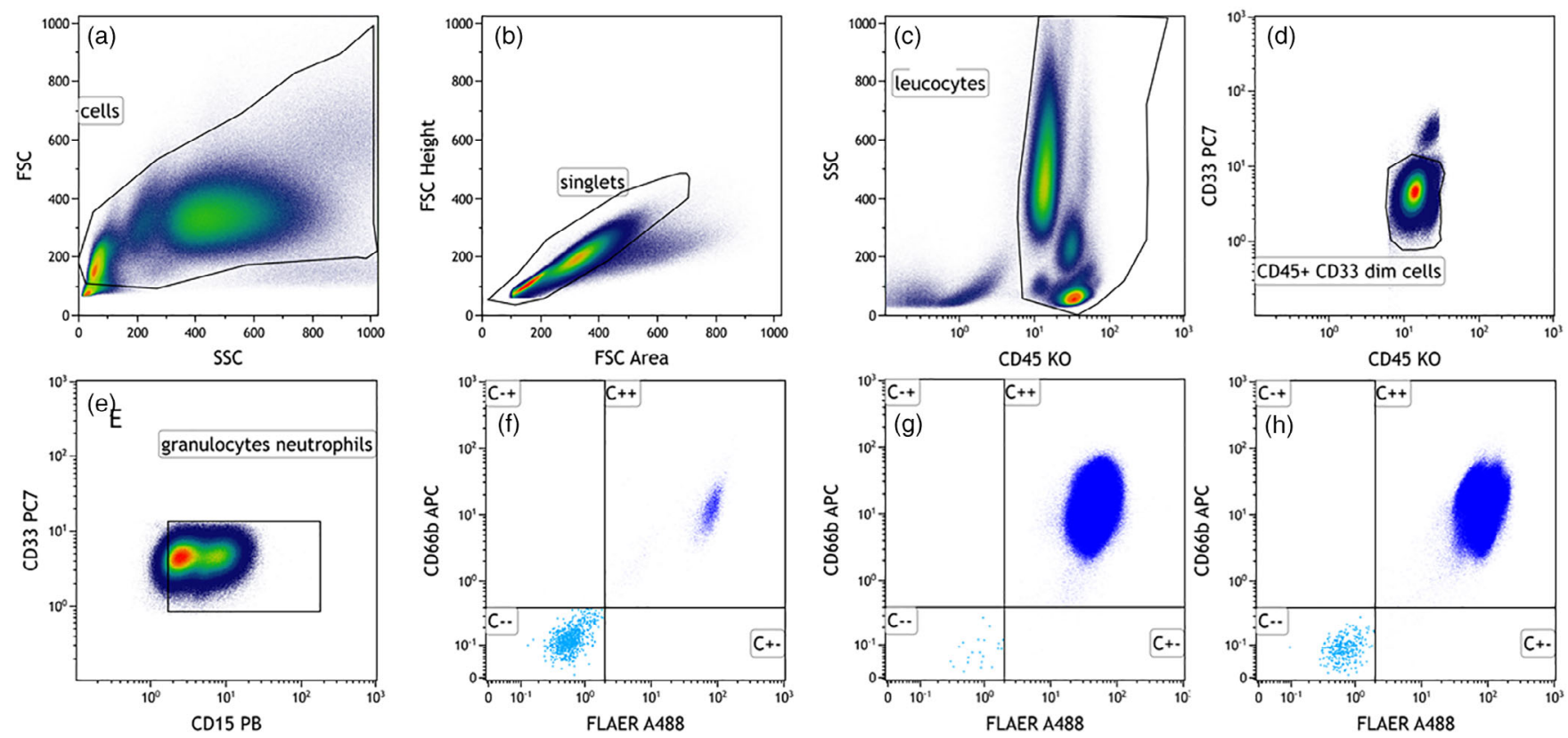

FIGURE 2 Gating strategy for GPI-deficient granulocyte analyses. (a) Cells initially identified by their forward scatter and side scatter profiles; (b) singlets identified by their forward scatter area and the forward scatter height; (c) CD45 positive cells were gated to select total leucocytes; (d) cells CD45-positive and CD33-dim were gated to coarsely select granulocytes among myeloid cells; (e) granulocytes were finally selected by gating the $95 \%$ brightest CD15 positive cells. Cells negative for both FLAER and CD66b fluorescence (C-quadrant) were considered GPI-deficient granulocytes whereas cells negative only for FLAER or only for CD66b were considered wild type granulocytes. F: PNH patient; G: healthy donor. $\mathrm{H}$ : cancer patient 
MF. Data concerning the repeatability criteria of the PIG-A assay during the TO sampling were analyzed using the Bland and Altman method, in which thresholds were set at $\pm 1.96 S D$ of the mean difference between duplicates. Exposure modalities were compared using Student's $t$ test. Assay results during exposure were compared using statistical tests for paired samples (Student or Wilcoxon tests, as appropriate). A two-sided value of $p<.05$ was considered statistically significant. Statistical analyses were performed using R software v2018 ("R foundation for statistical computing," Vienna, Austria) and GraphPad Prism version 8.0.0 for Windows (GraphPad Software, San Diego, CA).

TAB LE 1 Patient characteristics and sample results

\begin{tabular}{|c|c|c|c|c|c|c|c|c|c|c|c|c|c|c|}
\hline \multirow[b]{2}{*}{ Patients } & \multirow{2}{*}{$\begin{array}{l}\text { Age } \\
\text { (years) }\end{array}$} & \multirow[b]{2}{*}{ Group } & \multirow{2}{*}{$\begin{array}{l}\text { Dose } \\
\text { in PTV } \\
\text { (Gy) }\end{array}$} & \multirow[b]{2}{*}{ Chemotherapy } & \multirow{2}{*}{$\begin{array}{l}\text { Smoking } \\
\text { Status (PAU) }\end{array}$} & \multirow[b]{2}{*}{ TO } & \multicolumn{6}{|c|}{ (delay between timepoint and T0, days) } & \multicolumn{2}{|c|}{ CBML (\%o) } \\
\hline & & & & & & & T1 & & T2 & & T3 & & TO & T2 \\
\hline P1 & 67 & WBNRT & 65.1 & & NS & 3.3 & 0.8 & (21) & 11.7 & (42) & 2.4 & (325) & ND & ND \\
\hline P2 & 65 & WBRT & 66.5 & & NS & 12.4 & 3.5 & (21) & 29.5 & (49) & 28.9 & (224) & 4.5 & 5.5 \\
\hline P3 & 70 & WBRT & 66.4 & & NS & 2.3 & 4.7 & (21) & 4.2 & (49) & 2.7 & (178) & 9 & 20 \\
\hline P5 & 52 & WBNRT & 66.1 & & NS & 11.1 & 11.1 & (21) & 11.4 & (44) & 9.6 & (162) & ND & ND \\
\hline P6 & 48 & WBNRT & 50.1 & $\begin{array}{c}1 \text { (3 FEC + } 3 \\
\text { TXT) }\end{array}$ & NS & 16.1 & 6.2 & (21) & 7.7 & (35) & 0.6 & (171) & 8 & 43 \\
\hline P7 & 76 & WBRT & 66.6 & & NS & 2.2 & 2.2 & (22) & 1.6 & (49) & 2.1 & (393) & ND & ND \\
\hline P8 & 52 & WBRT & 66.8 & & NS & 8.1 & 3.1 & (20) & 14.5 & (48) & 12.3 & (201) & ND & ND \\
\hline P12 & 57 & WBNRT & 66.2 & & CS (10) & 3.1 & 2.5 & (21) & 1.7 & (47) & 2.1 & (343) & 3 & 25.5 \\
\hline P13 & 73 & WBRT & 66.4 & & NS & 0.8 & 0.7 & (21) & 2.6 & (45) & 2.6 & (303) & ND & ND \\
\hline P14 & 67 & WBRT & 66.3 & & NS & 0.0 & 0.0 & (21) & 0.4 & (50) & 0.0 & (282) & ND & ND \\
\hline P15 & 81 & WBNRT & 66.9 & & NS & 3.7 & 2.0 & (23) & 0.0 & (48) & ND & ND & 7.5 & 40 \\
\hline P16 & 65 & WBRT & 66.3 & & NS & 3.2 & 2.8 & (24) & 2.4 & (49) & 2.6 & (221) & 5 & 11.5 \\
\hline P17 & 59 & WBRT & 66.2 & & FS (30) & 2.8 & 2.8 & (22) & 4.2 & (46) & 1.6 & (177) & ND & ND \\
\hline P18 & 79 & WBNRT & 66.6 & & NS & 0.7 & 1.6 & (22) & 2.4 & (43) & 0.0 & (177) & 9 & 45 \\
\hline P19 & 48 & WBNRT & 66.6 & & CS (27) & 4.0 & 6.2 & (21) & 5.5 & (48) & 1.3 & (176) & 9.5 & 63.5 \\
\hline P26 & 38 & WBNRT & 60.1 & $\begin{array}{c}1(4 E C+9 \\
P T X)\end{array}$ & $\operatorname{CS}(6)$ & 8.2 & 6.1 & (22) & 4.7 & (42) & 0.0 & (143) & 4.5 & 31 \\
\hline P27 & 75 & WBNRT & 67.0 & & NS & 60.5 & 47.3 & (21) & 7.2 & (42) & 13.5 & (146) & ND & ND \\
\hline P28 & 52 & WBRT & 66.3 & 1 (6 FEC) & FS (15) & 6.6 & 4.7 & (20) & 5.7 & (45) & 0.8 & (121) & 9.5 & 31 \\
\hline P29 & 51 & WBNRT & 66.2 & $\begin{array}{c}1 \text { (3 FEC + } 9 \\
\text { PTX) }\end{array}$ & NS & 2.3 & 2.3 & (21) & 3.3 & (42) & 2.6 & (125) & ND & ND \\
\hline P30 & 51 & WBNRT & 66.4 & $\begin{array}{l}1 \text { (3 FEC } \\
\quad+3 T X T)\end{array}$ & NS & 4.4 & 2.1 & (21) & 2.2 & (42) & 0.4 & (131) & ND & ND \\
\hline
\end{tabular}

Note: Smoking status were described as non-smokers (NS), current (CS), and former smokers (FS) and quantified in pack-years unit (PAU). PIG-A MF were expressed per $10^{6}$. Average number of analyzed granulocytes in PIG-A assay was $2.7 \times 10^{6}$.

Abbreviations: C, Cyclophosphamide; CBML, cytokinesis-blocked micronucleated lymphocytes; E, Epirubicin; F, 5-fluoro-uracil; ND, not determined; PAU, pack-year unit; PTV, planning target volume; PTX, paclitaxel; TXT, docetaxel; WBNRT, whole breast and nodal radiation therapy; WBRT, whole breast radiation therapy. 


\section{3 | RESULTS}

\section{1 | Population description and sampling protocol}

Patient characteristics are summarized in Table 1. Fourteen (46.6\%) patients were included in the WBRT group, and 16 (53.3\%) were included in the WBNRT group. The mean age was $61.9( \pm 12.0)$ years. Five $(16.7 \%)$ patients received chemotherapy within 5 months before the initiation of RT. All five received a combination of epirubicin and cyclophosphamide. In addition, three received 5-fluorouracil, two received docetaxel, and two received paclitaxel. Eight (26.7\%) patients were smokers (five current smokers and three former smokers for over 2 years). None of the 30 patients were occupationally exposed to genotoxic agents. Within the 3 months preceding RT, 10 (33.3\%) patients had at least one X-ray examination, 4 (13.3\%) had a PET CT scan, 10 (33.3\%) had a bone scan, and $3(10.0 \%)$ had an infectious episode. No patient was occupationally exposed to ionizing radiation; one (3.3\%) reported asbestos exposure. The mean delay between the T0 and T1 blood samples was $21.6( \pm 2.5)$ days, that between the T0 and T2 blood samples was $44.5( \pm 4.5)$ days, and that between the T0 and T3 blood samples was 212.4 ( \pm 84.2 ) days (Table 1).

\section{2 | Delivered RT doses}

Delivered RT doses ranged from 50.1 to $66.9 \mathrm{~Gy}$, with a mean of 63.6 $( \pm 5.7) \mathrm{Gy}$. The proportion of the bone volume within the 0.5-Gy isodose volume was significantly higher in the WBNRT group than in the WBRT group: $589 \pm 101$ versus $233 \pm 96 \mathrm{~cm}^{3}(p<.001)$ (Table 2). However, there was no significant difference in the mean RT dose delivered to the bone between the patient groups: $8.5( \pm 1.4) \mathrm{Gy}$ versus $7.3( \pm 2.6) \mathrm{Gy}$, respectively.

\section{3 | Repeatability of the PIG-A assay}

T0 blood samples were tested in duplicate for each patient. Due to a technical issue in one duplicate, data were not available for four patients. Using a Bland and Altman plot, the technique revealed good repeatability.
Only $2 / 26$ (7.3\%) values exceeded the threshold set at \pm 1.96 SD around the mean of differences between each duplicate (Figure S2).

\subsection{Stability of samples over time}

TO blood samples from four patients were stored in the dark at $4^{\circ} \mathrm{C}$. The PIG-A assay was carried out at $0,24,48$, and $72 \mathrm{hr}$, and the

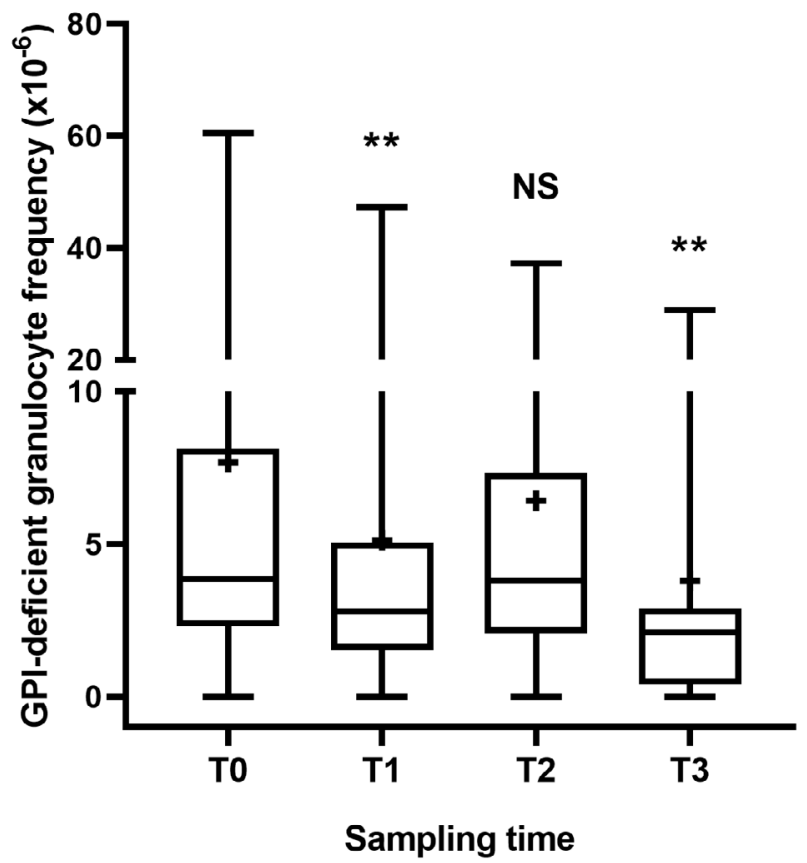

FIGURE 3 Overall GPI-deficient granulocyte frequencies before initiation of radiotherapy (TO), during (T1), at the end of treatment (T2) and during follow-up (T3) ( $n=30$, NS: non-significant; ${ }^{* *}: p<.01$ ). For each time point, lines are the median, boxes are the first and third quartile, whiskers are the minimum and maximum values and the plus sign is the mean. Despite very close data distributions (expressed as median [IQR], mean $\pm S D$, per million granulocytes), statistically significant decrease was noted between TO (3.9[2.3;8.2], $7.7 \pm 12.3$ ) and T1 (2.8[1.5;5.0], $5.1 \pm 8.7, p=.0027)$ and T0 and T3 (2.1[0.4;2.9], $3.8 \pm 6.2, p=.0049)$. No difference was observed between T0 and T2 (3.8[0.4;2.9], $6.4 \pm 8.2)$ distributions

TABLE 2 Irradiation data expressed as mean $\pm S D$

\begin{tabular}{|c|c|c|c|c|}
\hline & Overall $(n=30)$ & $\begin{array}{l}\text { Patients } \\
\text { WRRT }(n=14)\end{array}$ & WRNRT $(n=16)$ & \\
\hline & Overall $(n=30)$ & & WBNRI $(n=16)$ & $p$-value \\
\hline Bone volume at $0.5 \mathrm{~Gy}$ isodose $\left(\mathrm{cm}^{3}\right)$ & $423 \pm 205$ & $233 \pm 96$ & $589 \pm 101^{* * *}$ & $<.0001$ \\
\hline Minimal dose (Gy) & $0.41 \pm 0.07$ & $0.45 \pm 0.02$ & $0.36 \pm 0.06^{* * *}$ & $<.0001$ \\
\hline Mean dose (Gy) & $7.96 \pm 2.12$ & $7.35 \pm 2.64$ & $8.51 \pm 1.40^{\mathrm{ns}}$ & .15 \\
\hline Maximal dose (Gy) & $62.89 \pm 10.53$ & $64.33 \pm 5.94$ & $61.63 \pm 13.42^{\mathrm{ns}}$ & .47 \\
\hline
\end{tabular}

Note: For each patient, irradiated bone volume and doses were estimated with dosimetry calculations performed by the dedicated software of the treatment planning system. Significant variation was noted in the bone volume contained in the isodose $0.5 \mathrm{~Gy}$. Dose distribution was similar between groups.

Abbreviations: ns, non-significant; WBNRT: whole breast and nodal radiation therapy; WBRT, whole breast radiation therapy. ${ }^{* * *} p<.001$. 

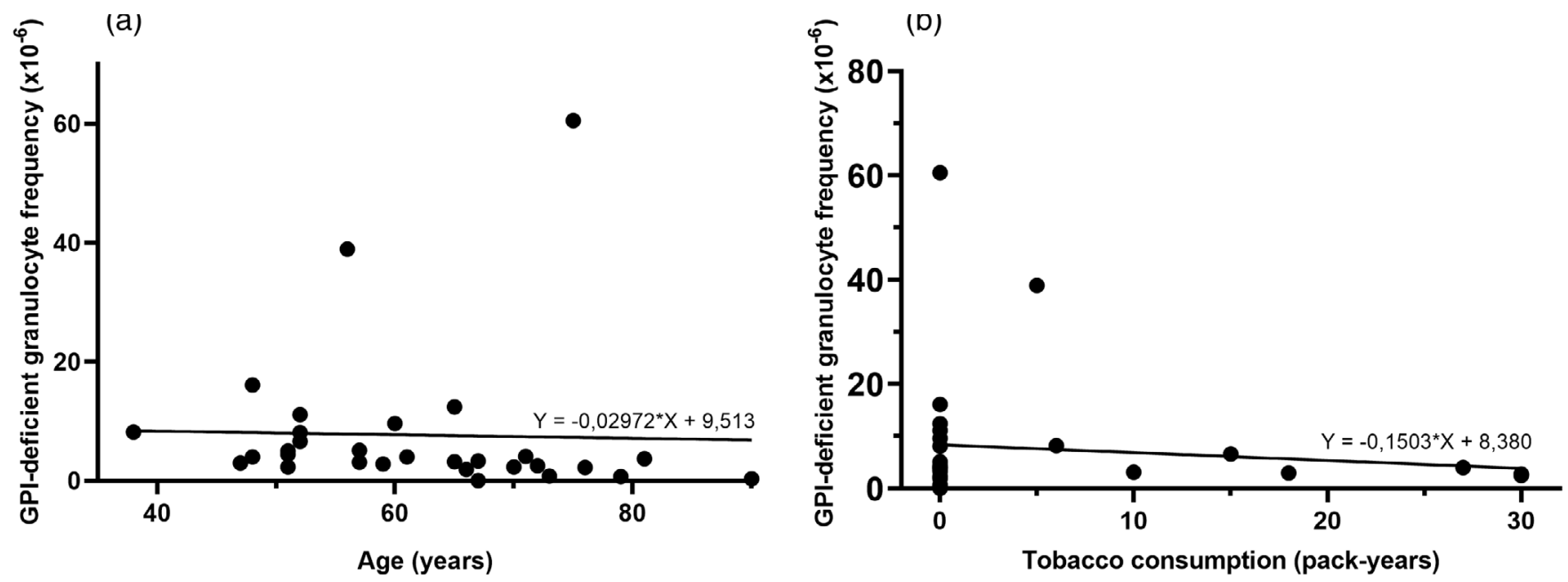

FIGURE 4 Impact of age (a) and smoking habits (b) on GPI-deficient granulocyte frequencies on T0 samples $(n=30)$. No relationship between GPI-deficient granulocyte frequencies and neither age nor tobacco consumption (expressed in pack year) was noted in the whole population

results were compared to the TO value generated at $0 \mathrm{hr}$ (Figure S3). No significant difference was found in the frequency of PIG-A mutant granulocytes between the 24,48 , and 72 -hr time points compared to the 0-hr time point.

\subsection{Effect of RT on the PIG-A MF}

The quantification of PIG-A-mutant granulocytes was performed in 30 patients before (T0), during (T1) and at the end of RT (T2). T3 samples were collected from 27 patients, as 3 patients declined to have the last sample taken. An average of $2.7( \pm 1.4)$ million granulocytes was counted per analysis. The mean $( \pm S D)$ PIG-A MFs were 7.7 per million $( \pm 12.1)$ at T0, $5.2( \pm 8.6)$ at T1, $6.4( \pm 8.0)$ at T2 and $3.8( \pm 36.0)$ at T3 (Figure 3). Although the data distributions at the different time points were extremely close, the frequency of PIG-A-mutant granulocytes was significantly lower at T1 $(p=.0035)$ and T3 ( $p=.006$ ) than at TO. No statistically significant difference in the PIGA MF was observed between TO and T2. Similar observations were noted within the WBRT and WBNRT groups (Figure S4A,B).

\section{6 | Effect of chemotherapy performed before the initiation of RT on the PIG-A MF}

Compared to patients not treated with chemotherapy, the PIG-A MF at TO was not significantly different in the five patients who had received chemotherapy before the initiation of RT (Table 1).

\section{7 | Effects of tobacco and age on the PIG-A MF}

Confounding factors represented by age and smoking status (current/ past or quantified in pack-years) were analyzed. None of these factors

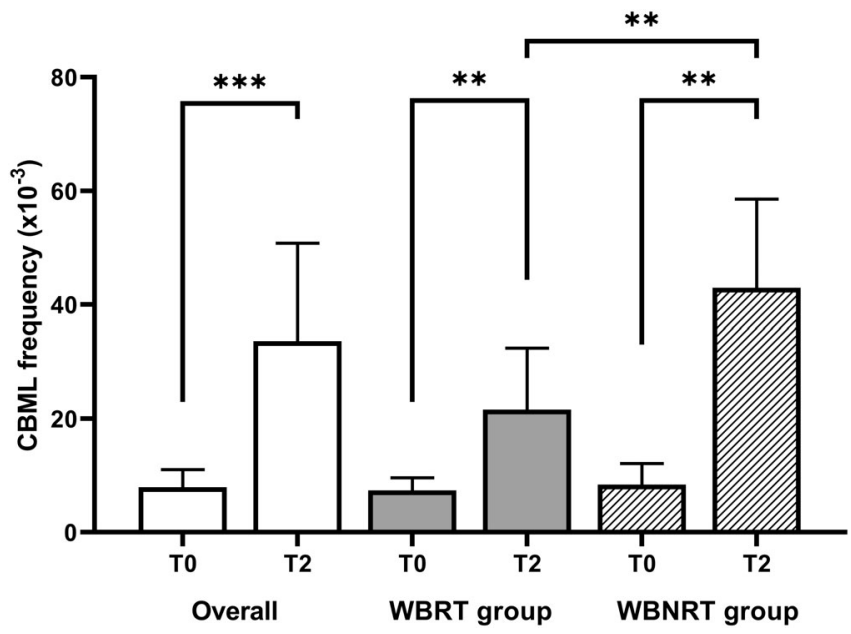

FIGURE 5 Distribution of CBML frequency per 1,000 cells before (TO) and at the end of radiotherapy (T2), for all patients ( $n=16)$ and in WBRT $(n=7)$ or WBNRT $(n=9)$ groups. Radiotherapy significantly increased CBML frequencies in overall, WBRT and WBNRT groups. At T2, CBML frequencies were statistically higher in WBNRT group than in WBRT group. ${ }^{*}: p<.05 ;^{* *}: p<.01 ;{ }^{* * *}: p<.001$. CBML, cytokinesis-blocked micronucleated lymphocytes; WBNRT, whole breast and nodal radiation therapy; WBRT, whole breast radiation therapy

were significantly associated with the PIG-A MF by univariate analysis (Figure 4a,b).

\section{8 | Effect of RT on CBML frequencies: The micronucleus test}

This test was performed with the T0 and T2 samples from 16 patients. The CBML frequency was significantly higher at T2 than at TO for all 
TAB LE 3 Identification of factors contributing to CBML values

\begin{tabular}{|llll|}
\hline $\begin{array}{l}\text { Genotoxic } \\
\text { endpoint }\end{array}$ & $\begin{array}{l}\text { Independent } \\
\text { variables }\end{array}$ & Coefficient & $\boldsymbol{p}$-value \\
\hline CBML & Radiotherapy group & 9.2 & .03 \\
& Smoking status & 8.2 & .11 \\
& Chemotherapy & 5.9 & .36 \\
\hline & $\begin{array}{l}\text { Radiotherapy } \\
\text { (TO vs. T2) }\end{array}$ & 25.7 & $<.0001$ \\
& Age & 0.4 & .043 \\
\hline
\end{tabular}

Note: The relative influence of the different independent variables (radiotherapy exposure categorized for all patients and according to WBRT or WBNRT group, smoking status categorized as smoking or nonsmoking, chemotherapy categorized as chemotherapy or no chemotherapy) on the CBML levels tested by multivariate linear regression analysis. Coefficients corresponds to the additional number of CBML predicted when the corresponding variable shows a one-unit shift or a category switch.

patients: $33.6( \pm 17.2)$ versus $7.9( \pm 3.2)(p<.001)$. The same differences were observed in the WBRT and WBNRT groups: $21.6( \pm 10.8)$ versus $7.4( \pm 2.2)(p=.0056)$ and $42.9( \pm 15.6)$ versus $8.4( \pm 3.7)$ $(p<.001)$, respectively (Figure 5$)$. The CBML frequency was significantly higher at T2 in the WBNRT group than in the WBRT group ( $p=.0083$ ). The CBML frequency at TO was not significantly influenced by previous administration of chemotherapy ( $p=.89$ ). By multivariate analysis, the CBML frequency was significantly correlated with age at TO $(p=.043)$ and irradiation extent at T2 $(p<.0001)$ but not with chemotherapy or the smoking status (Table 3). A significant association was found between age and the CMBL frequency at T0 $(p=.02)$ and between the CBML frequency at T2 and irradiation extent $(p=.02)$. No associations were found with chemotherapy or the smoking status (Table 3).

\section{4 | DISCUSSION}

To our knowledge, our study is the first to evaluate the value of the PIG-A gene mutation as a biomarker of genotoxicity in humans by detecting GPI-deficient human granulocytes before and after controlled genotoxic exposure. With a median PIG-A MF prior to any treatment of $3.9 \times 10^{-6}$ granulocytes (range: 0 to $60.5 \times 10^{-6}$ ), our results are similar to those published previously, supporting the validity of our method. Specifically, for a population of 142 healthy donors, Rondelli et al. reported a median frequency of PIG-A mutants of $4.9 \times 10^{-6}$ (range: $1 \times 10^{-6}$ to $37.5 \times 10^{-6}$ ) (Rondelli et al., 2013). The basal granulocyte PIG-A MFs in our study were consistent with previously published data on erythrocyte PIG-A MFs by Dertinger et al. and Cao et al. (Dertinger et al., 2015; Cao et al., 2016). Additionally, Horibata found similar basal PIG-A MFs in total red blood cells among healthy donors and cancer patients and, as we also reported, high spontaneous mutation frequencies for two patients (Horibata et al., 2016).

Our assay failed to demonstrate any increase in the PIG-A-mutant granulocyte population following RT in 30 patients recently diagnosed with breast cancer. The same lack of increase was also observed in the group with the largest irradiated bone volume (the WBNRT group). Several hypotheses may explain this absence of an increase in the PIG-A MF following RT. First, the bone marrow volume contained in the ribs and sternum represents 16.1 and $3.1 \%$ of the total hematopoietic bone marrow, respectively (Cristy, 1981). Since the irradiation administered in our RT protocol concerned only a part of the sternum and ribs, the probability of a granulocyte precursor gaining a mutation could have been too low to significantly impact the PIG-A MF observed in our population. Additionally, the high cytotoxic effect of RT should also be considered. Irradiations of more than $30 \mathrm{~Gy}$, even fractionated, have been described as cytotoxic for the bone marrow (Mauch et al., 1995). Due to damage to the medullary microenvironment, the recolonization of the bone marrow by hematopoietic stem cells after strong irradiation (>40 Gy) may take several years. Relatively high doses have been shown to cause irreversible injury to the microvasculature, leading to irrevocable bone marrow fibrosis (>50 Gy) (Mauch et al., 1995). It is therefore possible that the irradiated bone marrow may have been destroyed, resulting in the lack of increase in the PIG-A MF observed.

Granulocytes were chosen as the target human blood cells because they are unlikely to undergo complement-mediated lysis due to their short intravascular lifetime (Athens et al., 1961). It is unlikely that the absence of PIG-A-mutant granulocyte detection was related to technical issues. Indeed, our assay was directly inspired by the one routinely used for the diagnosis and monitoring of $\mathrm{PNH}$ patients (Sutherland et al., 2012). We aimed to optimize the phenotyping technique for circulating cells, while managing throughput and accuracy constraints and good repeatability of our assay. One should also consider the possibility that granulocytes were not the proper type of blood cells to target.

The fact that the micronucleus test was positive supports breast $\mathrm{RT}$ as a valuable genotoxic exposure model. CBML frequency is known as a reference assay for the assessment of genotoxic exposure. The increase in the CBML frequency observed after partial irradiation has been widely described in the literature (Thierens et al., 1995; Lee et al., 2002; Emamgholizadeh Minaei et al., 2016). In our study, increases in the CBML frequency were noted in both the WBRT and WBNRT groups following irradiation, and the induction of micronuclei seems to be correlated with the irradiated bone volume. The confirmation of the genotoxic nature of mammary irradiation by the micronucleus test performed with lymphocytes suggests an inability of our assay to demonstrate an increase in the PIG-A MF in granulocytes. However, the positivity of CBMLs in lymphocytes may not be inconsistent with the absence of PIG-A mutation induction in granulocytes. Lymphocytes, being circulating cells, likely had a shorter exposure time to RT and therefore were exposed to a lower radiation dose than myeloid hematopoietic stem cells, which are precursors of granulocytes, contained in the irradiated medullary stroma. As mentioned above, high doses of radiotherapy (>30 Gy) could have led to bone marrow fibrosis and stem cell apoptosis.

Moreover, lymphocytes were sampled just before and within $2 \mathrm{hr}$ after the end of the irradiation regimen and forced to divide ex vivo 
so that DNA double-strand breaks, which are known to be induced by ionizing radiation, induced micronuclei formation. In contrast, to produce an increase in the PIG-A MF, mutations must be fixed in the bone marrow via cell division, and the cells bearing mutations must transit to the peripheral blood. One cannot exclude that the turnover of granulocytes may not have been sufficient for a small cohort of mutated cells to significantly affect the PIG-A MF. Myeloid precursors perpetually undergo mitosis and thus are probably more sensitive to genotoxic stimuli than circulating lymphocytes, which are quiescently maintained in the GO phase of the cell cycle. Indeed, because of the long circulating lifespan of lymphocytes, chromosomal damage can be revealed later by an ex vivo culture step. The persistence of CBMLs, which has been reported up to 5 years after irradiation, could reflect this clinical feature (Lee et al., 2002). Finally, ionizing radiation is among the most potent clastogens and therefore may be poorly detected by an X-linked reporter gene mutation. The CBML frequency is increased following chromosome breakage and/or losses, whereas PIG-A MFs should be mainly increased following gene mutation; thus, the nature of the DNA and/or chromosomal damage could have an impact on the sensitivities of the two assays. The accurate examination of the individual evolution of the PIG-A MF during RT showed three recognizable patterns: no modification of the PIG-A MF while the CBML frequency increased (12 patients), an increase in the PIG-A MF only (1 patient) and increases in both the PIG-A MF and the CBML frequency ( 3 patients). Even if the small sample size does not allow a definitive conclusion to be reached, interindividual differences in response to mutagen and clastogen exposures may explain these findings.

To our knowledge, only four studies have already evaluated the consequences of ionizing radiation and/or chemotherapy on the frequency of PIG-A-mutant cells in human blood. MacDiarmid et al. did not observe any increase in the PIG-A-mutant lymphocyte frequency following depleted uranium exposure in military veterans (McDiarmid et al., 2011). Horibata et al evaluated the PIG-A assay with human red blood cells using a cohort of 27 cancer patients treated with chemotherapy and/or RT. The authors did not report any increase in the PIG-A-mutant cell frequency among the patients treated with RT ( $n=14$ ) (Horibata et al., 2016). Nevertheless, this population was highly heterogeneous, with different types of cancers, various RT modalities and possibly unsuitable sampling times. Dobrovolsky et al evaluated the PIG-A assay with red blood cells in a cohort of 10 cancer patients treated with chemotherapy. Six of the patients had breast cancer and received chemotherapeutic agents similar to those used in our study. Using this cell type as a target, they found only an approximately three-fold increase in the frequency of red blood cells originating from a PIG-A-mutant precursor in a single patient treated with a combination of cisplatin and etoposide (Dobrovolsky et al., 2011). In contrast, Haboubi et al. recently found that the PIG-A assay performed with erythrocytes could help to detect esophageal adenocarcinoma and might be a useful predictor of disease progression (Haboubi et al., 2019). It may thus be possible that the sensitivity of the PIG-A assay differs depending on the origin of genomic instability.

\section{5 | CONCLUSION}

Our assay failed to demonstrate an increase in the PIG-A-mutant granulocyte population following RT for breast cancer therapy, even in the group with the largest irradiated bone volume (WBNRT group). Further studies are needed to evaluate the sensitivity of the PIG-A assay in humans subjected to genotoxic exposures with targeting of different blood cells.

\section{ACKNOWLEDGMENTS}

This work was completed with the support of (a) the A*MIDEX project "CREER" (No. ANR-11-IDEX-0001-02) funded by the "Investissements d'Avenir," French Government Program of the French National Research Agency (ANR); (b) SATT-SE (ID 870); and (c) ARS PACA (REVELA13; dossier N. 20120658). The authors would like to thank Dr. Romaric Lacroix and Dr. Sylvie Cointe for helpful discussion and Jocelyne Pompili for helpful technical assistance.

\section{AUTHOR CONTRIBUTIONS}

Xavier Carcopino: Designed the study and applied for Research Ethics Board approval; approved the final manuscript. Pierre Castel: Designed the study and applied for Research Ethics Board approval; collected the data; analyzed the data and prepared draft figures and tables; prepared the manuscript draft with important intellectual input from X. Carcopino, S. P. Robert, V. M. Tassistro, C. A. Delecourt, and T. G. Orsière; approved the final manuscript; had complete access to the study data. Didier Cowen: Designed the study and applied for Research Ethics Board approval; recruited the patients; approved the final manuscript. Thierry G. Orsière: Designed the study and applied for Research Ethics Board approval; collected the data; approved the final manuscript; had complete access to the study data. Rémi M. Bonetto: Recruited the patients; analyzed the data and prepared draft figures and tables; prepared the manuscript draft with important intellectual input from X. Carcopino, S. P. Robert, V. M. Tassistro, C. A. Delecourt, and T. G. Orsière; approved the final manuscript; had complete access to the study data. Stéphane P. Robert: Collected the data; analyzed the data and prepared draft figures and tables; approved the final manuscript; had complete access to the study data. Virginie M. Tassistro: Collected the data; analyzed the data and prepared draft figures and tables; approved the final manuscript. Camille A. Delecourt: Analyzed the data and prepared draft figures and tables; approved the final manuscript. Magalie Claeys-Bruno: Analyzed the data and prepared draft figures and tables; approved the final manuscript. Michelle Sergent: Analyzed the data and prepared draft figures and tables; approved the final manuscript.

\section{REFERENCES}

Araten, D.J., Nafa, K., Pakdeesuwan, K. and Luzzatto, L. (1999) Clonal populations of hematopoietic cells with paroxysmal nocturnal hemoglobinuria genotype and phenotype are present in normal individuals. 
Proceedings of the National Academy of Sciences of the United States of America, 96, 5209-5214.

Athens, J.W., Haab, O.P., Raab, S.O., Mauer, A.M., Ashenbrucker, H., Cartwright, G.E. and Wintrobe, M.M. (1961) Leukokinetic studies. IV. The total blood, circulating and marginal granulocyte pools and the granulocyte turnover rate in normal subjects. The Journal of Clinical Investigation, 40, 989-995.

Bhalli, J.A., Pearce, M.G., Dobrovolsky, V.N. and Heflich, R.H. (2011) Manifestation and persistence of pig-a mutant red blood cells in C57BL/6 mice following single and split doses of $\mathrm{N}$-ethyl-N-nitrosourea. Environmental and Molecular Mutagenesis, 52, 766-773.

Borowitz, M.J., Craig, F.E., Digiuseppe, J.A., Illingworth, A.J., Rosse, W., Sutherland, D.R., Wittwer, C.T., Richards, S.J. and Clinical Cytometry Society. (2010) Guidelines for the diagnosis and monitoring of paroxysmal nocturnal hemoglobinuria and related disorders by flow cytometry. Cytometry. Part B, Clinical Cytometry, 78, 211-230.

Brodsky, R.A. (2014) Paroxysmal nocturnal hemoglobinuria. Blood, 124 2804-2811.

Cao, Y., Yang, L., Feng, N., Shi, O., Xi, J., You, X., Yin, C., Yang, H., Horibata, K., Honma, M., Qian, B., Weng, W. and Luan, Y. (2016) A population study using the human erythrocyte PIG-A assay: erythrocyte PIG-A assay-based population study. Environmental and Molecular Mutagenesis, 57, 605-614.

Cao, Y., Wang, T., Xi, J., Zhang, G., Wang, T., Liu, W., You, X., Zhang, X., Xia, Z. and Luan, Y. (2020b) PIG-A gene mutation as a genotoxicity biomarker in human population studies: an investigation in leadexposed workers. Environmental and Molecular Mutagenesis, 61, 611-621.

Cao, Y., Wang, X., Liu, W., Feng, N., Xi, J., You, X., Chen, R., Zhang, X., Liu, Z. and Luan, Y. (2020a) The potential application of human PIG-A assay on azathioprine-treated inflammatory bowel disease patients. Environmental and Molecular Mutagenesis, 61, 456-464.

Cristy, M. (1981) Active bone marrow distribution as a function of age in humans. Physics in Medicine and Biology, 26, 389-400.

Dertinger, S.D., Avlasevich, S.L., Bemis, J.C., Chen, Y. and MacGregor, J.T. (2015) Human erythrocyte PIG-A assay: an easily monitored index of gene mutation requiring low volume blood samples: human erythrocyte PIG-A assay. Environmental and Molecular Mutagenesis, 56, 366-377.

Dertinger, S.D., Avlasevich, S.L., Torous, D.K., Bemis, J.C., Phonethepswath, S., Labash, C., Carlson, K., Mereness, J., Cottom, J., Palis, J. and MacGregor, J.T. (2014) Persistence of cisplatin-induced mutagenicity in hematopoietic stem cells: implications for secondary cancer risk following chemotherapy. Toxicological Sciences: An Official Journal of the Society of Toxicology, 140, 307-314.

Dobrovolsky, V.N., Elespuru, R.K., Bigger, C.A.H., Robison, T.W. and Heflich, R.H. (2011) Monitoring humans for somatic mutation in the endogenous PIG-a gene using red blood cells. Environmental and Molecular Mutagenesis, 52, 784-794.

Emamgholizadeh Minaei, S., Mozdarani, H., Motazakker, M., Mansouri, M. and R Aghamiri, S.M. (2016) Evaluation of cytogenetic alterations in peripheral blood lymphocytes of esophageal cancer patients treated with radiotherapy or Chemoradiotherapy using cytokinesis-blocked micronucleus assay. Acta Medica Iranica, 54, 9-14.

Fenech, M. (2007) Cytokinesis-block micronucleus cytome assay. Nature Protocols, 2, 1084-1104.

Gollapudi, B.B., Lynch, A.M., Heflich, R.H., Dertinger, S.D., Dobrovolsky, V. N., Froetschl, R., Horibata, K., Kenyon, M.O., Kimoto, T., Lovell, D.P., Stankowski, L.F., White, P.A., Witt, K.L. and Tanir, J.Y. (2015) The in vivo pig-a assay: a report of the International Workshop On Genotoxicity Testing (IWGT) Workgroup. Mutation Research. Genetic Toxicology and Environmental Mutagenesis, 783, 23-35.

Graupner, A., Eide, D.M., Instanes, C., Andersen, J.M., Brede, D.A., Dertinger, S.D., Lind, O.C., Brandt-Kjelsen, A., Bjerke, H., Salbu, B., Oughton, D., Brunborg, G. and Olsen, A.K. (2016) Gamma radiation at a human relevant low dose rate is genotoxic in mice. Scientific Reports, 6, 32977.

Haboubi, H.N., Lawrence, R.L., Rees, B., Williams, L., Manson, J.M., AlMossawi, N., Bodger, O., Griffiths, P., Thornton, C. and Jenkins, G.J. (2019) Developing a blood-based gene mutation assay as a novel biomarker for oesophageal adenocarcinoma. Scientific Reports, 9, 5168.

Horibata, K., Ukai, A., Ishikawa, S., Sugano, A. and Honma, M. (2016) Monitoring genotoxicity in patients receiving chemotherapy for cancer: application of the PIG-A assay. Mutation Research. Genetic Toxicology and Environmental Mutagenesis, 808, 20-26.

Houtgraaf, J.H., Versmissen, J. and van der Giessen, W.J. (2006) A concise review of DNA damage checkpoints and repair in mammalian cells. Cardiovascular Revascularization Medicine: Including Molecular Interventions, 7, 165-172.

Hu, R., Mukhina, G.L., Piantadosi, S., Barber, J.P., Jones, R.J. and Brodsky, R.A. (2005) PIG-A mutations in normal hematopoiesis. Blood, 105, 3848-3854.

Kawagoe, K., Takeda, J., Endo, Y. and Kinoshita, T. (1994) Molecular cloning of murine pig-a, a gene for GPI-anchor biosynthesis, and demonstration of interspecies conservation of its structure, function, and genetic locus. Genomics, 23, 566-574.

Lee, T.-K., Allison, R.R., O'Brien, K.F., Naves, J.L., Karlsson, U.L. and Wiley, A.L. (2002) Persistence of micronuclei in lymphocytes of cancer patients after radiotherapy. Radiation Research, 157, 678-684.

Mauch, P., Constine, L., Greenberger, J., Knospe, W., Sullivan, J., Liesveld, J.L. and Deeg, H.J. (1995) Hematopoietic stem cell compartment: acute and late effects of radiation therapy and chemotherapy. International Journal of Radiation Oncology, Biology, Physics, 31, 1319-1339.

McDiarmid, M.A., Albertini, R.J., Tucker, J.D., Vacek, P.M., Carter, E.W., Bakhmutsky, M.V., Oliver, M.S., Engelhardt, S.M. and Squibb, K.S. (2011) Measures of genotoxicity in gulf war I veterans exposed to depleted uranium. Environmental and Molecular Mutagenesis, 52, 569-581.

Miura, D., Dobrovolsky, V.N., Kimoto, T., Kasahara, Y. and Heflich, R.H. (2009) Accumulation and persistence of pig-a mutant peripheral red blood cells following treatment of rats with single and split doses of N-ethyl-N-nitrosourea. Mutation Research, 677, 86-92.

Ohtani, S., Unno, A., Ushiyama, A., Kimoto, T., Miura, D. and Kunugita, N. (2012) The in vivo pig-a gene mutation assay is useful for evaluating the genotoxicity of ionizing radiation in mice. Environmental and Molecular Mutagenesis, 53, 579-588.

Ohtani, S., Ushiyama, A., Ootsuyama, A. and Kunugita, N. (2014) Persistence of red blood cells with pig-a mutation in p53 knockout mice exposed to $\mathrm{X}$-irradiation. The Journal of Toxicological Sciences, 39, 7-14.

Olsen, A.-K., Dertinger, S.D., Krüger, C.T., Eide, D.M., Instanes, C., Brunborg, G., Hartwig, A. and Graupner, A. (2017) The pig-a gene mutation assay in mice and human cells: a review. Basic \& Clinical Pharmacology \& Toxicology, 121(Suppl 3), 78-92.

Parker, C., Omine, M., Richards, S., Nishimura, J.-I., Bessler, M., Ware, R., Hillmen, P., Luzzatto, L., Young, N., Kinoshita, T., Rosse, W., Socié, G. and International PNH Interest Group. (2005) Diagnosis and management of paroxysmal nocturnal hemoglobinuria. Blood, 106, 3699-3709.

Peruzzi, B., Araten, D.J., Notaro, R. and Luzzatto, L. (2010) The use of PIG-A as a sentinel gene for the study of the somatic mutation rate and of mutagenic agents in vivo. Mutation Research, 705, 3-10.

Phonethepswath, S., Bryce, S.M., Bemis, J.C. and Dertinger, S.D. (2008) Erythrocyte-based pig-a gene mutation assay: demonstration of crossspecies potential. Mutation Research, 657, 122-126.

Phonethepswath, S., Franklin, D., Torous, D.K., Bryce, S.M., Bemis, J.C., Raja, S., Avlasevich, S., Weller, P., Hyrien, O., Palis, J., Macgregor, J.T. and Dertinger, S.D. (2010) Pig-a mutation: kinetics in rat erythrocytes 
following exposure to five prototypical mutagens. Toxicological Sciences: An Official Journal of the Society of Toxicology, 114, 59-70.

Rondelli, T., Berardi, M., Peruzzi, B., Boni, L., Caporale, R., Dolara, P., Notaro, R. and Luzzatto, L. (2013) The frequency of granulocytes with spontaneous somatic mutations: a wide distribution in a normal human population. PloS One, 8, e54046.

Sutherland, D.R., Keeney, M. and Illingworth, A. (2012) Practical guidelines for the high-sensitivity detection and monitoring of paroxysmal nocturnal hemoglobinuria clones by flow cytometry. Cytometry Part B: Clinical Cytometry, 82B, 195-208.

Takeda, J., Miyata, T., Kawagoe, K., lida, Y., Endo, Y., Fujita, T., Takahashi, M., Kitani, T. and Kinoshita, T. (1993) Deficiency of the GPI anchor caused by a somatic mutation of the PIG-A gene in paroxysmal nocturnal hemoglobinuria. Cell, 73, 703-711.

Thierens, H., Vral, A., Van Eijkeren, M., Speleman, F. and De Ridder, L. (1995) Micronucleus induction in peripheral blood lymphocytes of patients under radiotherapy treatment for cervical cancer or Hodgkin's disease. International Journal of Radiation Biology, 67, 529-539.

Torous, D.K., Avlasevich, S.L., Khattab, M.G., Baig, A., Saubermann, L.J., Chen, Y., Bemis, J.C., Lovell, D.P., Walker, V.E., MacGregor, J.T. and Dertinger, S.D. (2020) Human blood PIG-A mutation and micronucleated reticulocyte flow cytometric assays: method optimization and evaluation of intra- and inter-subject variation. Environmental and Molecular Mutagenesis, 61, 807-819.

UNEP. (2016) Radiation effects and sources: What is radiation? What does radiation do to us? Where does radiation come from? . Wien, Austria: UNEP.

United Nations. (2000) Sources and effects of ionizing radiation: United Nations scientific committee on the effects of atomic radiation: UNSCEAR 2000 report to the general assembly, with scientific annexes. New York, NY: United Nations. 